\title{
BENCHMARKING STIFF ODE SOLVERS FOR ATMOSPHERIC CHEMISTRY PROBLEMS II: ROSENBROCK SOLVERS
}

\author{
A. SANDU \\ Program in Applied Mathematics and Computational Sciences, The University of Iowa, lowa City, \\ IA 52242, U.S.A. \\ J. G. VERWER, J. G. BLOM and E. J. SPEE \\ Center for Mathematics and Computer Science (CWI), P.O. Box 94079. 1090 GB, Amsterdam, Netherlands

\section{G. R. CARMICHAEL} \\ Center for Global and Regional Environmental Research, The University of Iowa, Iowa City, IA 52242. \\ U.S.A. \\ and
}

\section{F. A. POTRA}

Department of Mathematics, The University of Iowa, Iowa City, IA 52242, U.S.A.

(First received 25 July 1996 and in final form 10 January 1997. Published August 1997)

\begin{abstract}
In the numerical simulation of atmospheric transport-chemistry processes, a major task is the integration of the stiff systems of ordinary differential equations describing the chemical transformations. It is therefore of interest to systematically search for stiff solvers which can be identified as close to optimal for atmospheric applications. In this paper we continue our investigation from Sandu et al. (1996, CWI Report NM-R9603 and Report in Comput. Math., No. 85) and compare eight solvers on a set of seven box-models used in present day models. The focus is on Rosenbrock solvers. These turn out to be very well suited for our application when they are provided with highly efficient sparse matrix techniques to economize on the linear algebra. Two of the Rosenbrock solvers tested are from the literature, viz. RODAS and ROS4, and two are new and specially developed for air quality applications, viz. RODAS3 and ROS3. (O) 1997 Elsevier Science Ltd.
\end{abstract}

Key word index: Atmospheric chemistry, air pollution modeling, numerical stiff ODEs, Rosenbrock methods, sparsity.

\section{INTRODUCTION}

To better understand the transport and fate of trace gases and pollutants in the atmosphere, comprehensive air quality models have been developed. For their numerical solution, very often the operator splitting approach is followed. A major computational task is then the numerical integration of the stiff ODE (ordinary differential equation) systems describing the chemical transformations. This integration must be carried out repeatedly at all spatial grid points for all split intervals chosen, so that the model runs readily require an enormous amount of integrations. It is therefore of interest to systematically search for stiff ODE solvers which for atmospheric applications can be identified as close to optimal. In this paper we continue our search from
(Sandu et al., 1996b), where a large number of box-model tests were carried out with nine solvers. Among these were dedicated explicit methods and general purpose solvers from the numerical stiff $O D E$ field, all provided with sparse matrix techniques to economize on the numerical algebra operations. Three main conclusions were drawn in (Sandu et al., 1996b):

- All sparse general solvers work quite efficiently on all test problems, although their ranking relative to each other depends on the test problem. Compared were the BDF (Backward Differentiation) solvers VODE (Brown et al., 1989) and LSODES (Hindmarsh, 1983), the Runge-Kutta solver SDIRK4 (Hairer and Wanner, 1991) and the Rosenbrock solver RODAS (Hairer and Wanner, 1991). 
- TWOSTEP (Verwer, 1994; Verwer et al., 1996b) is by far the best within the class of dedicated explicit methods. It outperforms a number of QSSA solvers, often by a wide margin. However, it is in general less efficient than sparse implicit solvers. The code is advocated for gas-phase problems only and, like all other dedicated explicit solvers tested, not capable of treating gas-liquid phase chemistry.

- Sparse RODAS is competitive to all solvers tested and often is the fastest for low to moderate accuracies.

RODAS partly owes its competitiveness to its onestep nature. This is important in view of the large number of restarts carried out in the box-model runs. Restarts must be considered because the solvers are examined for application in an operator splitting approach. The multistep BDF (Gear) codes are then less attractive since their growth in step size is limited by stability considerations.

Our experience with RODAS is in line with results from (Hairer and Wanner, 1991), where for a number of stiff ODEs from other applications RODAS was shown to be competitive with other solvers for low to modest accuracies. Because for atmospheric applications the greatest interest lies in high efficiency for very low accuracy (two figures at most), it is worthwhile to continue our search within the class of Rosenbrock methods. Thus, the aim of this paper is to assess whether other Rosenbrock solvers can be found which, for our specific purpose, constitute an improvement over RODAS in terms of efficiency.

The paper is organized as follows. In Section 2 we briefly review our test set from (Sandu et al., 1996b) and describe a new test problem. This test problem is also solved with the EBI (Euler Backward Iterative) method proposed in Hertel et al. (1993). Section 3 contains a brief introduction to Rosenbrock methods, put together for the convenience of readers from the atmospheric research community. An appendix to this section is added for those readers who wish to learn more on Rosenbrock methods. In Section 4 we discuss all eight solvers which were tested. These include the two Rosenbrock solvers RODAS and ROS4 from (Hairer and Wanner, 1991) and two new Rosenbrock solvers which were developed for this benchmark, viz. RODAS3 and ROS3. The special purpose solver EBI from Hertel et al. (1993) was applied to the first test problem only, since it is dependent on the chemical mechanism. For the purpose of a wider comparison we also present results for the extrapolation code SEULEX from Hairer and Wanner (1991) and for TWOSTEP and VODE. The latter two were also tested in Sandu et al. (1996b). Section 5 describes the set up of the experiments and Section 6 contains all the test results. The final section summarizes the main conclusions.

To enable interested readers to further extend this benchmark comparison using their own solvers, as well as to extend our problem set with other challenging example problems from atmospheric chemistry, all the software we have used for the problems and the solvers have been put on the ftp-site (CGRER ftp site, 1996).

\section{THE BENCHMARK PROBLEMS}

The test set used in this paper consists of seven box-model problems. Except for number one, i.e. Problem A, all remaining problems, i.e. Problems B-G, are almost identical to those used in Sandu et al. (1996b). To save space we therefore present B-G only very briefly and refer to Sandu et al. (1996b) for a complete description of these models. All problems were run for five days. This time interval is sufficiently large for taking into account several diurnal cycles of the photochemical reactions. The unit of time is seconds and the unit for the concentrations is number of molecules per $\mathrm{cm}^{3}$. The problems were uniformly coded in FORTRAN using the symbolic preprocessor KPP (Damian-Iordache and Sandu, 1995). This uniformity is important for a meaningful intercomparison. An exception exists for problem A. The FORTRAN program of the EBI implementation for this problem was obtained from Dentener $(1993,1996)$. Tables containing initial concentrations and emission values of the most important species can be found in Sandu et al. (1996b).

Problem A: The TMk model. The problem was borrowed from Dentener $(1993,1996)$. It describes the reduced $\mathrm{CH}_{4} / \mathrm{CO} / \mathrm{HO}_{x} / \mathrm{NO}_{x}$ chemistry and is used in the global dispersion model TMk (Heimann, 1983). It consists of 36 reactions between 18 species of which 2 were held fixed, namely $\mathrm{H}_{2} \mathrm{O}$ and $\mathrm{O}_{2}$. Since new values of the photolysis rates are available every $40 \mathrm{~min}$, we split accordingly the five day period (see Section 5 for more details). The simulated conditions correspond to a polluted air parcel in summer time, at $45^{\circ}$ north latitude and at ground level (pressure $=1000 \mathrm{mbar}$ ). We have included emissions of NO at a constant level of $10^{6} \mathrm{mlc} \mathrm{cm}^{-3} \mathrm{~s}^{-1}$. More information about this model can be found in Dentener (1993). We note that for this small problem (17 components) the exploitation of sparsity results in limited benefits. The Jacobian matrix has 90 non-zero entries and 93 after the factorization.

Problems $B$ and C: The CBM-IV model. These are based on the Carbon Bond Mechanism IV (Gery et al., 1989) consisting of 32 chemical species involved in 70 thermal and 11 photolytic reactions. Test problem $B$ describes an urban scenario and simulates a heavily polluted atmosphere. Test problem $C$ describes a rural atmosphere.

Problems D and E: The AL model. Problems D and $\mathrm{E}$ employ the kinetic mechanism that is presently used in the STEM-II model (Carmichael et al., 1986), consisting of 84 non-constant chemical species involved in 142 thermal and 36 photolytic reactions. 
The mechanism, based on the work of Lurmann et al. (1986) and Atkinson et al. (1989), can be used to study the chemistry of both highly polluted (e.g. near urban centers) and remote (e.g. marine) environments. Problem $D$ describes an urban scenario and problem $\mathrm{E}$ a rural one. The simulated conditions are identical to those employed in problems B and C, respectively.

Problem F: A stratospheric model. This test problem is based on the chemical mechanism that was used in the NASA HSRP/AESA stratospheric models intercomparison. The initial concentrations and the values of the rate constants follow the NASA region A scenario. There are 34 non-constant species involved in 84 thermal and 25 photolytic reactions. No emissions were prescribed.

Problem G: An aqueous model. This aqueous chemistry model contains 65 non-constant species involved in 77 thermal and 11 photolytic gas-phase chemical reactions, 39 liquid-phase chemical reactions and 39 gas-liquid mass transfer reactions. The gas-phase mechanism is based on CBM-IV, while the liquid-phase mechanism is based on a chemical scheme the authors obtained from Matthijsen (1995). All dedicated explicit solvers tested in Sandu et al. (1996b) failed on this problem.

\section{ROSENBROCK METHODS}

This section is devoted to a brief introduction to Rosenbrock methods, put together for the convenience of readers from the atmospheric research community. Part of the notation has been adopted from Hairer and Wanner (1991), where Rosenbrock methods are described in much greater detail (Sections IV.7, IV.10 and VI.3). An introductory appendix has been added for those readers who wish to learn more about the theory behind Rosenbrock methods.

\subsection{The integration formula}

Rosenbrock methods are usually considered in conjunction with stiff ODE systems in the autonomous form

$$
\dot{y}=f(y), \quad t>t_{0}, \quad y\left(t_{0}\right)=y_{0}
$$

This places no restriction since every non-autonomous system $\dot{y}=f(t, y)$ can be put in the form (1) by treating time $t$ also as a dependent variable, i.e. by augmenting the system with the equation $t=1$. In atmospheric applications it is often the case that the reaction coefficients are held constant on each split step interval; the chemical rate equations obtained this way are in autonomous form.

Usually stiff ODE solvers use some form of implicitness in the discretization formula for reasons of numerical stability. The simplest implicit scheme is the backward Euler method

$$
y_{n+1}=y_{n}+h f\left(y_{n+1}\right)
$$

where $h=t_{n+1}-t_{n}$ is the step size and $y_{n}$ the approximation to $y(t)$ at time $t=t_{n}$. Since $y_{n+1}$ is defined implicitly, this numerical solution itself must also be approximated. Usually some modification of the iterative Newton method is used, again for reasons of numerical stability. Suppose that just one iteration per time step is applied. If we then assume that $y_{n}$ is used as the initial iterate, the following numerical result is found

$$
\begin{aligned}
y_{n+1} & =y_{n}+k, \\
k & =h f\left(y_{n}\right)+h J k,
\end{aligned}
$$

where $J$ denotes the Jacobian matrix $f^{\prime}\left(y_{n}\right)$ of the vector function $f$.

The numerical solution is now effectively computed by solving the system of linear algebraic equations that defines the increment vector $k$, rather than a system of nonlinear equations. Rosenbrock (1963) proposed to generalize this linearly implicit approach to methods using more stages, so as to achieve a higher order of consistency. The crucial consideration put forth was to no longer use the iterative Newton method, but instead to derive stable formulas by working the Jacobian matrix directly into the integration formula. His idea has found widespread use and a generally accepted formula (Hairer and Wanner, 1991) for a so-called s-stage Rosenbrock method, is

$$
\begin{gathered}
y_{n+1}=y_{n}+\sum_{i=1}^{s} b_{i} k_{i} \\
k_{i}=h f\left(y_{n}+\sum_{j=1}^{i-1} x_{i j} k_{j}\right)+h J \sum_{j=1}^{i} \gamma_{i j} k_{j}
\end{gathered}
$$

where $s$ and the formula coefficients $b_{i}, \alpha_{i j}$ and $\gamma_{i j}$ are chosen to obtain a desired order of consistency and stability for stiff problems. An introduction on the properties of consistency, stability and stiff accuracy for Rosenbrock methods is presented in an appendix.

For a reason explained later, the coefficients $\gamma_{i i}$ are taken equal for all stages, i.e. $\gamma_{i i}=\gamma$ for all $i=1, \ldots, s$. For $s=1, \gamma=1$ the above linearized implicit Euler formula is recovered. For the non-autonomous system $\dot{y}=f(t, y)$, the definition of $k_{i}$ is changed to

$$
\begin{aligned}
k_{i}= & h f\left(t_{n}+\alpha_{i} h, y_{n}+\sum_{j=1}^{i-1} \alpha_{i j} k_{j}\right)+\gamma_{i} h^{2} \frac{\partial f}{\partial t}\left(t_{n}, y_{n}\right) \\
& +h J \sum_{j=1}^{i} \gamma_{i j} k_{j}
\end{aligned}
$$

where

$$
x_{i}=\sum_{j=1}^{i-1} \alpha_{i j}, \quad \gamma_{i}=\sum_{j=1}^{i} \gamma_{i j}
$$


Like Runge-Kutta methods, Rosenbrock methods successively form intermediate results

$$
Y_{i}=y_{n}+\sum_{j=1}^{i-1} \alpha_{i j} k_{j}, \quad 1 \leqslant i \leqslant s,
$$

which approximate the solution at the intermediate time points $t_{n}+\alpha_{i} h$. Rosenbrock methods are therefore also called Runge-Kutta-Rosenbrock methods. Observe that if we identify $J$ with the zero matrix and omit the $\partial f / \partial t$ term, a classical explicit Runge-Kutta method results.

Rosenbrock methods are attractive for a number of reasons. Like fully implicit methods, they preserve exact conservation properties due to the use of the analytic Jacobian matrix. However, they do not require an iteration procedure as for truly implicit methods and are therefore more easy to implement. They can be developed to possess optimal linear stability properties for stiff problems. They are of one-step type, and thus can rapidly change step size. We recall that this is of particular importance for our application in view of the many operator-split restarts.

\subsection{Reducing computational costs}

Each time step requires an evaluation of the Jacobian $J, s$ matrix-vector multiplications with $J$ and, assuming that $\gamma_{i i}=\gamma, s$ solutions of a linear system with (the same) matrix $I-\gamma h J$, accompanied with $s$ derivative evaluations. The multiplications with $J$ are easily avoided in the actual implementation by a simple transformation (see Section IV.7 of Hairer and Wanner, 1991). Because of the multistage nature, the computational costs for a Rosenbrock method, spent within one time step, are often considered to be high compared to the costs of say a linear multistep method of the BDF type. In particular, the Jacobian update and the solution of the $s$ linear systems, requiring one matrix factorization (LU-decomposition) and $s$ backsolves (forward-backward substitutions) typically account for most of the CPU time used by a Rosenbrock method. On the other hand, if a Rosenbrock code solves the problem efficiently in fewer steps than a BDF code needs, then the CPU time for a whole integration using a Rosenbrock method can become significantly less then for a BDF method.

Sparsity. For large atmospheric chemistry models the number of zeroes in $J$ readily amounts to $\approx 90 \%$. This high level of sparsity can be exploited to signifcantly reduce the costs of the linear algebra calculations. For this task we use the symbolic preprocessor KPP (Damian-Iordache and Sandu, 1995). KPP prepares a sparse matrix factorization with only a minimal fill-in (see Table 1 in Sandu et al., 1996a) and delivers a FORTRAN routine for the backsolve without indirect addressing. Altogether this means that the numerical algebra is handled very efficiently. The sparse matrix technique implemented in KPP is based on a diagonal Markowitz criterion (see Damian-Iordache and Sandu (1995) and Sandu et al. (1996a) for more details).

Approximate Jacobians. It is conceivable to attempt to further reduce the numerical algebra costs through an approximate Jacobian.

- One possibility is to use a time-lagged Jacobian $J=f^{\prime}\left(y_{n+\eta}\right)$ where $\eta=0,-1, \ldots$ such that $n+\eta$ is constant. If we define $J$ this way, and in addition keep $h$ fixed, then $I-\gamma h J$ is a constant matrix during the number of times that the parameter $\eta$ is decreased; hence one can advance several time steps using the same LU-decomposition. The derivation of order conditions (which circumvents the order reduction associated with the time-lagging of the Jacobian) can be found in Verwer and Scholz (1983) and Verwer et al. (1983b). Since the exact Jacobians are used, conservation properties will still be maintained.

- Replacing $J$ by a matrix with a simpler structure, say a matrix of higher sparsity, may result in further savings in linear algebra costs, but will destroy the conservation properties. Also, the number of order conditions will significantly increase (see the Wmethods of Steihaug and Wolfbrandt, 1979).

- One can devise methods based on a partitioning of the species into slow and fast ones where part of the entries of $J$ is put to zero. This approach does not maintain conservation properties either and adds the problem of devising a good partitioning strategy.

In the current paper the above ideas are not explored: only exact Jacobians are considered.

\subsection{Step-size control}

General purpose stiff oDE solvers normally adapt the step size in an automatic manner to enable small step sizes at times when the solution gradients are large and large step sizes when solution gradients are small. For Runge-Kutta solvers an effective and simple step size control can be based on a so-called embedded formula

$$
\tilde{y}_{n+1}=y_{n}+\sum_{i=1}^{s} \tilde{b}_{i} k_{i},
$$

which uses the already computed increment vectors $k_{i}$. The approximation $\tilde{y}_{n+1}$ thus differs only in the choice of the weights $\tilde{b}_{i}$ and hence is available at no extra costs. Usually, the weights are chosen such that the order of consistency of $\tilde{y}_{n+1}$ is $\tilde{p}=p-1$, if $p$ is the order of $y_{n+1}$. This suggests to use the difference vector $E s t=\tilde{y}_{n+1}-y_{n+1}$ as a local error estimator. In what follows we will denote the order of such a pair of formulas by $p(\tilde{p})$. All the Rosenbrock solvers (RODAS, RODAS3, ROS4 and ROS3) use embedded formulas to estimate the local error.

The specific step size strategy goes as follows. Let $m$ denote the dimension of the ODE system. Let 
$\operatorname{Tol}_{k}=$ atol + rtol $\left|y_{n+1, k}\right|$, where atol and rtol represent a user-specified absolute and relative error tolerance and $y_{n+1, k}$ the $k$-th component of $y_{n+1}$. Tolerances may differ componentwise, but are here taken equal for all components for simplicity of testing. Denote

$$
\operatorname{Err}=\sqrt{\frac{1}{m} \sum_{k=1}^{m}\left(\frac{\text { Est }_{k}}{\text { Tol }_{k}}\right)^{2}}
$$

The integration step is accepted if $\mathrm{Err}<1$ and rejected otherwise and redone. The step size for the new step, both in the rejected and accepted case, is estimated by the usual step size prediction formula

$$
h_{\text {new }}=h \min \left(10, \max \left(0.1,0.9 /(\operatorname{Err})^{1 /(\tilde{p}+1)}\right)\right) .
$$

At the first step after a rejection, the maximal growth factor of 10 is set to 1.0. Further, $h$ is constrained by a minimum $h_{\min }$ and a maximum $h_{\max }$ and at any start of the integration for each operator-split interval we begin with a starting step size $h=h_{\text {start }}$. A rejection of the first step is followed by a ten times reduction of $h$. These step size constraints will be specified later. Because the maximal growth factor is equal to 10 , the step size adjusts very rapidly and quickly attains large values if the solution is sufficiently smooth and $h=h_{\text {start }}$ is chosen small.

\section{THE SOLVERS}

In this section we list all solvers which have been tested. The solvers RODAS3 and ROS3 are new. For these we give the defining formula coefficients. All other solvers are existing ones and are described only briefly. The Rosenbrock solvers have order of consistency 3 or 4. Preliminary experiments with two second order solvers, based on Method III from Verwer (1977) and on the complex-valued method from Rosenbrock (1963) (advocated in Dnestrovskaya et al., 1994) gave disappointing results.

RODAS: This Rosenbrock solver from Hairer and Wanner (1991) is based on a stiffly accurate pair of order $4(3)$. Both formulas are L-stable. The number of stages $s$ equals six and also six derivative evaluations and six backsolves are used. In Sandu et al. (1996b) RODAS was one of the best solvers tested.

RODAS 4: This Rosenbrock solver is also taken from Hairer and Wanner (1991). It implements a number of 4-stage 4(3) pairs which all require four derivative evaluations and four backsolves. Hence, per step ROS4 is somewhat cheaper than RODAS. However, in Hairer and Wanner (1991) a comparison is presented favouring RODAS, which is attributed to the stiff accuracy property (the methods of ROS4 are not stiffly accurate). We have tested its L-stable version (see Table 7.2, in Hairer and Wanner, 1991) and found that generally its performance was very close to that of ROS3 and RODAS3. We therefore decided to omit presenting results for ROS4.

RODAS 3: The third Rosenbrock solver was designed along the same principles as RODAS. It is based on a stiffly accurate, embedded pair of order 3(2). The number of stages is $s=4$, requiring four backsolves but only three derivative evaluations are used. Hence per step it needs less work than RODAS, but it is one order lower. We have selected this pair since we aim at optimal efficiency for low accuracies. To the best of our knowledge, this pair of formulas has not yet been proposed elsewhere. The coefficients $x_{i j}$ and $\gamma_{i j}$ are

$$
\begin{aligned}
\left(x_{i j}\right) & =\left(\begin{array}{llll}
0 & & & \\
0 & 0 & & \\
1 & 0 & 0 & \\
3 / 4 & -1 / 4 & 1 / 2 & 0
\end{array}\right), \\
\left(\gamma_{i j}\right) & =\left(\begin{array}{llll}
1 / 2 & \\
1 & 1 / 2 & & \\
-1 / 4 & -1 / 4 & 1 / 2 & \\
1 / 12 & 1 / 12 & -2 / 3 & 1 / 2
\end{array}\right)
\end{aligned}
$$

and the weights are

$$
\begin{gathered}
\left(b_{i}\right)=\left(\begin{array}{lll}
5 / 6-1 / 6-1 / 6 & 1 / 2
\end{array}\right), \\
\left(\tilde{b}_{i}\right)=\left(\begin{array}{lll}
3 / 4-1 / 4 & 1 / 2 & 0
\end{array}\right)
\end{gathered}
$$

Both formulas are L-stable. Observe that the embedded one is defined by the final intermediate approximation $Y_{4}$. The values of $\gamma$ for L-stability are presented in Table 1.

ROS 3: The fourth Rosenbrock solver is based on an embedded pair of order 3(2) and is also new. The number of stages is $s=3$ involving three backsolves and two derivative evaluations. The third order method is L-stable and the embedded second order method is strongly A-stable $(R(\propto)=0.5)$. The stiff accuracy property is not valid for ROS3. The method was constructed under the design criteria: order three, L-stability for both the stability function and the internal stability functions, and a strongly A-stable second order embedding. The internal stability functions are associated with the intermediate approximations (5). Imposing stability for these internal functions was advocated in Verwer (1977) as a means to improve a Rosenbrock method for strongly nonlinear stiff problems. We note in passing that if the order

Table 1. Values of $\gamma$ for L-stability

\begin{tabular}{lll}
\hline$s$ & L-stability, $p \geqslant s-1$ & L-stability, $p=s$ \\
\hline 1 & & $\gamma=1$ \\
2 & $(2-\sqrt{2}) / 2 \leqslant \gamma \leqslant(2+\sqrt{2}) / 2$ & $\gamma=(2 \pm \sqrt{2}) / 2$ \\
3 & $0.18042531 \leqslant \gamma \leqslant 2.18560010$ & $\gamma=0.43586652$ \\
4 & $0.22364780 \leqslant \gamma \leqslant 0.57281606$ & $\gamma=0.57281606$ \\
\hline
\end{tabular}


of consistency equals 3 and $s=3$, then the requirement of L-stability prevents the existence of an Lstable second order embedding. The coefficients are

$$
\begin{aligned}
\gamma & =0.43586652150845899941601945119356 \\
\gamma_{21} & =-0.19294655696029095575009695436041 \\
\gamma_{32} & =1.74927148125794685173529749738960 \\
b_{1} & =-0.75457412385404315829818998646589 \\
b_{2} & =1.94100407061964420292840123379419 \\
b_{3} & =-0.18642994676560104463021124732829 \\
\sigma_{1} & =-1.53358745784149585370766523913002 \\
b_{2} & =2.81745131148625772213931745457622 \\
\sigma_{3} & =-0.28386385364476186843165221544619 .
\end{aligned}
$$

The remaining coefficients are $\alpha_{21}=\alpha_{31}=\gamma$ and $\alpha_{32}=\gamma_{31}=0$.

VODE. This solver from Brown et al. (1989) is a general purpose BDF Gear code and can be regarded as a successor of LSODE (Hindmarsh, 1983), which is popular in the field of atmospheric chemistry as a reference code. In Sandu et al. (1996b) voDE performed satisfactorily and we include it again for comparison with the Rosenbrock solvers. VODE uses the same sparsity routines as the Rosenbrock solvers.

TWOSTEP. This solver from Verwer (1994), Verwer and Simpson (1995), and Verwer et al. (1996b) is based on the second order BDF formula and uses, instead of the usual modified Newton method, a Gauss-Seidel or Jacobi iteration for approximately solving the implicit BDF relations. In the tests of this paper only the Gauss-Seidel iteration is used. It was developed as a special purpose, explicit solver for atmospheric chemistry problems. In Sandu et al. (1996b) it outperforms a number of solvers based on the QSSA approach. We include it again for comparison with the Rosenbrock solvers. The same implementation as in Sandu et al. (1996b) is used, which always performs two Gauss-Seidel iterations and automatically adjusts the step size.

SEULEX. The solver SEULEX is also taken from Hairer and Wanner (1991). It bears a relationship with the Rosenbrock solvers, as it builds up a solution from the (non-autonomous) linearly implicit Euler method, i.e., $y_{n+1}=y_{n}+(I-h J)^{-1} h f\left(t_{n}, y_{n}\right)$, by Richardson extrapolation. The use of this Euler method in an extrapolation code for stiff ODEs was first suggested in Deuflhard (1985) A rule of thumb is that the virtue of extrapolation manifests itself most clearly when high accuracy is required (see also Hairer and Wanner, 1991). We have included SEULEX in our benchmarking as the extrapolation approach is mentioned by Zlatev (1995) (see Section 3.4.3) as a viable one for atmospheric ODE problems, although no results seem to have been reported yet. The same sparse linear algebra as used for the other solvers was implemented. The extrapolation sequence defined by iwork $(4)=4$ was used. This sequence was found to work well for our application. Other settings are given default values.

EBI. The Euler backward iterative (EBI) method was proposed by Hertel et al. (1993). Being based on the Euler backward implicit formula (2), its main feature is that, instead of using Newton's method, the implicit solution is approximated through a semianalytical, problem dependent iteration process. This process groups species together which allow an exact solution of the implicit equations after putting part of them at the old time level. Species equations which do not fit in an appropriate grouping are treated with a form of Jacobi iteration. Satisfactory results are reported (Hertel et al., 1993) for different scenario's based on the CBM-IV mechanism. The approach can also be applied when using higher BDF methods since use of these implicit methods leads to a similar system of equations, but a considerable drawback is that the iterative solution method is adapted to the particular chemistry scheme. We therefore have tested the method only for the TMk model, using an implementation obtained from Dentener (1996). This implementation contains no local error control mechanism so that constant step sizes are taken.

\section{SET-UP OF EXPERIMENTS}

Splitting interval. The tests are intended to simulate an operator splitting environment. In air quality models, most often a symmetric splitting is used, for example:

$$
T_{x}^{\Delta t} \circ T_{y}^{\Delta t} \circ T_{z}^{\Delta t} \circ C^{2 \Delta t} \circ T_{z}^{\Delta t} \circ T_{y}^{\Delta t} \circ T_{x}^{\Delta t}
$$

where $T_{j}^{\Delta t}$ stands for transport in direction $j$ for a time interval $\Delta t$ and $C$ is the chemistry solution operator. Thus the restart time or splitting interval equals $2 \Delta t$. For Problem A we have chosen a restart time of $40 \mathrm{~min}$ and for all other problems $60 \mathrm{~min}$. A restart time of $60 \mathrm{~min}$ corresponds to a transport step size of $30 \mathrm{~min}$ due to the symmetry of splitting. For the two urban scenarios (test problems $B$ and D) additional simulations were carried out with a restart each $15 \mathrm{~min}$; this corresponds to a splitting interval of $7.5 \mathrm{~min}$ for the transport scheme.

Emissions. For all problems except the stratospheric problem $F$, emissions are prescribed. In the experiments we have computed emissions at the beginning of each split interval, simulating a form of operator splitting. This means that species solutions for which emissions occur, are made discontinuous so that at any restart initial transients occur. We thus simulate, to some extent, what happens in a true transport computation where one also encounters initial transients at any restart. As a rule, strong initial transients make the nonlinear stiff problems harder to solve. If we would compute the emissions along with the integration over the split intervals, then all species solutions remain continuous at restart.

Steering parameters. For variable step size solvers the important steering parameters are $h_{\text {starl }}, h_{\min }$ and the local error tolerances atol, rtol. A user-specified $h_{\min }$ is important. Without a prescribed minimum, step sizes can result as 
small as the shortest time constants, sometimes even $\approx 10^{-8}-10^{-9} \mathrm{~s}$. Step size values close to these extremely short time constants are redundant, since the minimal time constants of importance for photochemical models lie between $1 \mathrm{~s}$ and $1 \mathrm{~min}$, approximately. On the temporal scale of interest, species with a smaller time constant quickly reach their (solution dependent) steady state when they are perturbed. On the other hand, most solvers require a relatively small step size at the start to resolve the initial transients.

Through trial and error we have prescribed the following values for $h_{\min }$ and $h_{\text {mar }}$ which are imposed for all solvers (except EBI): for the tropospheric Problems A-E, $h_{\min }=0.1 \mathrm{~s}$ and $h_{\text {stan }}=60 \mathrm{~s}$; for the stratospheric Problem F, $h_{\text {min }}=h_{\text {start }}=0.001 \mathrm{~s}$; and for the aqueous Problem G $h_{\min }=h_{\text {star }}=0.0001 \mathrm{~s}$. These values concern the $1 \mathrm{~h}$ restart time. For the tropospheric problems B, D with the $15 \mathrm{~min}$ restart time, we have taken $h_{\text {start }}=h_{\min }=0.1 \mathrm{~s}$.

For all problems and all solvers except EBL, we have prescribed the absolute tolerance value atol $=0.01 \mathrm{mlcm}^{-3}$ along with a sequence of relative tolerance values rtol such that effectively relative local error control is imposed. For a given method, the different data points in the accuracy-efficiency plots correspond to this sequence. The values used are

rtol $=1.0,3.0 \times 10^{-1}, 1.0 \times 10^{-1}, 3.0 \times 10^{-2}, 1.0 \times 10^{-2}$,

$$
3.0 \times 10^{-3}, 1.0 \times 10^{-3}, 3.0 \times 10^{-4}, 1.0 \times 10^{-4} \text {. }
$$

Needless to mention that the actual resulting accuracies are always different from the given local tolerances. The tolerances merely govern the local error and step size control. Also note that for very large values of rtol, say rtol $>0.1$, the control is very loose so that a negative number of significan digits (6) or even a breakdown may be the result. Note that a negative number of significant digits (6) means relative errors greater than $100 \%$. The Rosenbrock solvers RODAS, ROS3 and the BDF solver VODE showed breakdowns more often, RODAS 3 only for $\mathrm{rtol}=1$, while SEULEX never failed and always returned a positive SDA. Also TwOSTEP never encountered a breakdown, only minor negative SDA values. Data points corresponding to a breakdown or a negative result, as well as points with SDA $>4$ or with an exceptionally large CPU time have been skipped from the plots. We have used a wide range of tolerances merely for illustrative purposes.

Accuracy. The numerical results were compared to a very accurate reference solution (given by RADAUS, $\mathrm{rtol}=10^{-12}$, componentwise set atol) using a temporal modified root mean square norm of the relative error. With the reference solution $y$ and the numerical solution $\hat{y}$ available at $\left\{t_{n}=t_{0}+n \Delta t, 0 \leqslant n \leqslant N\right\}$, we first compute for each species

$$
\mathrm{ER}_{k}=\sqrt{\frac{1}{\left|f_{k}\right|} \cdot \sum_{n \in r_{1}}\left|\frac{y_{k}\left(t_{n}\right)-\hat{y}_{k}\left(t_{n}\right)}{y_{k}\left(t_{n}\right)}\right|^{2}},
$$

where $f_{k}=\left\{0 \leqslant n \leqslant N: y_{k}\left(t_{n}\right) \geqslant a\right\}$. This value is then represented in the plots through the number of significant digits for the maximum of $\mathrm{ER}_{k}$, defined by

$$
S D A=-\log _{10}\left(\max _{k} E_{k}\right) .
$$

Note that if the set $\mathscr{I}_{k}$ is empty for a chosen threshold $a$, the value of $E_{R_{k}}$ is neglected. This threshold factor serves to eliminate chemically meaningless large relative errors for concentration values smaller than $a \mathrm{mlc} \mathrm{cm}^{-3}$ in the error measure. We used $a=10^{6} \mathrm{mlc} \mathrm{cm}^{-3}$ for all tropospheric problems and $a=10^{4} \mathrm{mlc} \mathrm{cm}{ }^{-3}$ for the stratospheric one. Additional experiments performed with $a=1 \mathrm{mlc} \mathrm{cm}-3$ led to nearly the same conclusions. In all plots presented in the remainder for problems B-F, including those for the $15 \mathrm{~min}$ restart times for $\mathrm{B}, \mathrm{D}$, we have used $N=120$. So we always sample at the endpoint of each hour over the whole 5 days. For Problem A we sample at the end of every $40 \mathrm{~min}$. Observe that $\mathrm{SDA}=2$ means $1 \%$ accuracy in the error measure used. In discussing the results in the next section we focus on this accuracy level.

Timing. The answer to the question of which method is "the fastest" may depend also on the machine. In order to measure the influence of the hardware on the relative performance of integrators we have performed all the numerical experiments on two completely different architectures, namely a HP.UX 935 A workstation (double precision, $\approx 14$ digits) and a Cray C98 (scalar mode, single precision, $\approx 14$ digits); in addition, some of the experiments were also repeated on a SGI workstation (double precision, $\approx 14$ digits). Very similar results were found. As a consequence, in what follows only the HP work-precision diagrams are presented. We plot the SDA values against efficiency, i.e. the measured CPU times on a logarithmic scale in unit seconds.

Reaction coefficients. In practice the rate coefficients can be implemented in two ways, either as time-continuous functions or as functions piecewise constant per split interval The time-continuous function implementation of the thermal rate coefficients may lead to a large number of exponential function evaluations per time step, which are very costly. For example, with Rosenbrock methods we observed that these calculations can be as expensive as the sparse matrix factorization. Since for the actual practice true time dependency seems redundant, we have used piecewise constant rate coefficients per operator-split subinterval (temperature and solar angle frozen using values halfway). Observe that in (Sandu et al., 1996b) time-continuous values were used. We did again a number of tests with time-continuous values in the current investigation but observed no notable differences in the relative performances of the solvers.

\section{RESULTS AND ILLUSTRATIONS}

\subsection{Problem A: the TMk model}

The work precision diagram is given in Fig. 1. Results are presented for all the solvers discussed above, including EBI. The EBI results are obtained with constant step sizes of length

$$
\begin{aligned}
h= & 40 / 80,40 / 40,40 / 20,40 / 10,40 / 6,40 / 5,40 / 4, \\
& 40 / 3 \mathrm{~min} .
\end{aligned}
$$

The number of iterations within EBI was in all runs equal to 8 (cf. Dentener, 1996). The results show that the variable step size Rosenbrock solvers are clearly superior to all others for $1 \%$ accuracy. SEULEX appears to be faster than VODE, but slower than the Rosenbrock codes. However, the gap between these solvers decreases for higher accuracies. Among the Rosenbrock codes, RODAS3 and ROS3 have similar performance in the low accuracy domain; they are followed closely by RODAS. EBI and TWOSTEP perform reliably but cannot compete with RODAS3 over the whole accuracy range.

\subsection{Problems $B$ and $C$ : the $C B M-I V$ model}

In Fig. 2 the results for test problems $B(1 \mathrm{~h}$ restart time) and $C$ are presented. For the rural problem all Rosenbrock solvers perform equally well, 


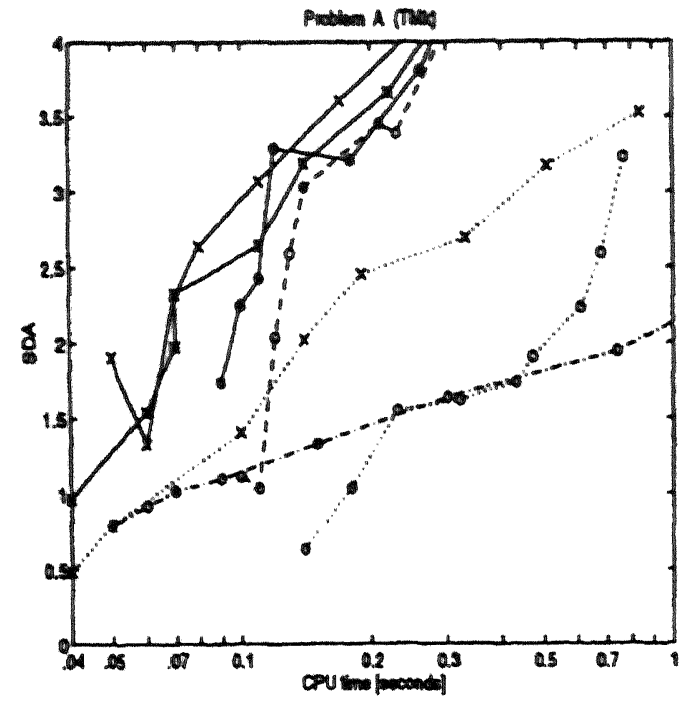

Fig. 1. Work-precision diagram for test problem A (TMk): Sparse RODAs3 (solid line with "*"), Sparse ROS3 (solid line with " $x$ "), Sparse RODAs (solid line with " 0 "), TwOSTEP SEIDEL. (dotted line with " $x$ "), Sparse vODE (dotted line with " 0 "), Sparse SEULEX (dashed line with "O") and EBI (dashdotted line with "o").

followed by SEULEX, while VODE and TWOSTEP fall behind. This also holds for the urban problem, but now a distinction exists between RODAS3, ROS 3 and SEULEX, RODAS. Up to about 3 digits RODAS 3 and ROS3 perform best. For accuracies higher than 3 digits RODAS takes over.

The results for the urban problem with a $15 \mathrm{~min}$ restart time are presented in Fig. 3. The relative per- formances between the solvers remains almost the same. The main difference with the $1 \mathrm{~h}$ restart time is seen in the CPU times. All integrations become roughly 3 to 4 times more expensive, showing that all solvers spend most of their time in the start-up phase. Recall that the start-up phase has become longer as we have lowered $h_{\text {alart }}$ from $60 \mathrm{~s}$ to $h_{\text {min }}=0.1 \mathrm{~s}$. The $60 \mathrm{~s}$ starting step size was found too large for the Rosenbrock solvers for a good performance. This indicates that they must spent quite an effort in resolving the initial transients. However, they remain competitive, in particular ROS3 and RODAS3. The figure also contains results for the most simple QSSA solver we previously applied in Sandu et al. (1996b). However, this solver again lags behind to all others.

\subsection{Problems $D$ and $E$ : the $A L$ model}

For problems $D$ and $E$ with $1 \mathrm{~h}$ restart time the results are given in Fig. 4. It is interesting to compare code performances to those obtained for the CBM-IV model since the same urban and rural scenarios are simulated. They differ, however, in the number of species and reactions, the AL model being considerably larger. For the urban problem RODAS3 and ROS3 are again the fastest, up to 3 digits, while for higher accuracies RODAS becomes better. SEULEX now performs somewhat less than for the CBM-IV model, whereas TWOSTEP is notably better positioned. In the rural case all solvers perform close, except VODE; both in the rural and urban case vODE falls behind. Notable is the close performance of ROS3 and RODAS3. As a general conclusion, Rosenbrock codes are again superior to the BDF ones. The better relative
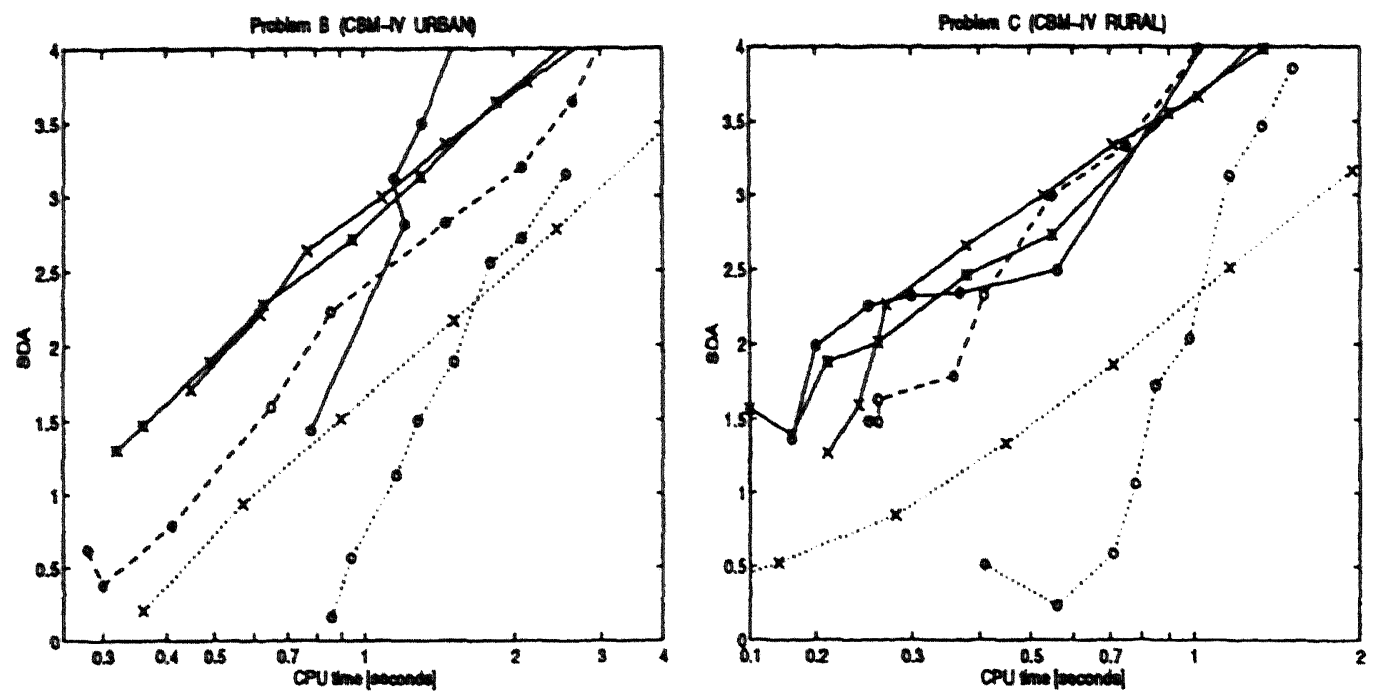

Fig. 2. Work-precision diagram for test problems B and C (CBM-IV): Sparse RODAs3 (solid line with "*n), Sparse ROS3 (solid line with " $x$ "), Sparse RODAS (solid line with " 0 "), rwOSTEP SEIDEL (dotted line with " $x$ ") Sparse VODE (dotted line with "o") and Sparse SEULEX (dashed line with "o"). 

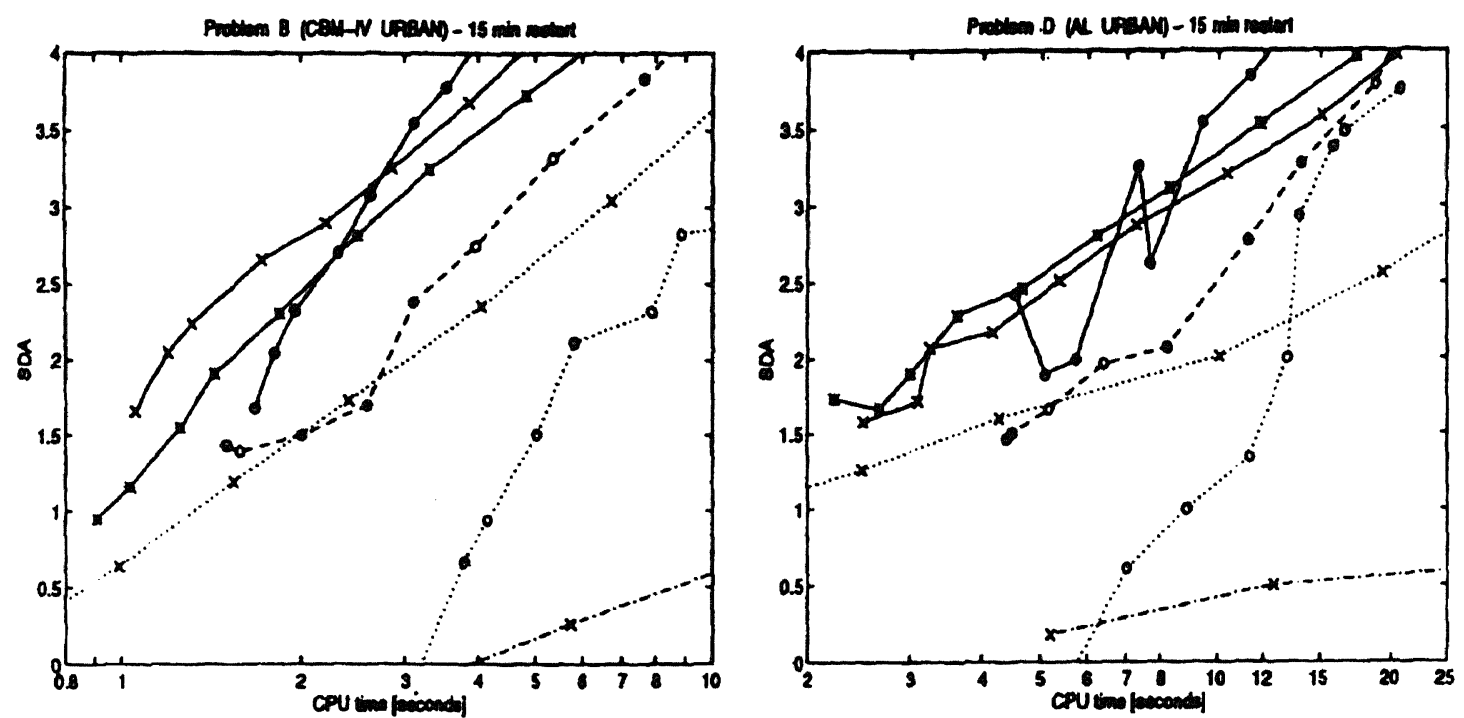

Fig. 3. Work-precision diagram for test problems B (CBM-IV urban) and D (AL urban), with a restart each 15 min: Sparse RODAS 3 (solid line with "*"), Sparse ROS3 (solid line with " $x$ "), Sparse RODAS (solid line with “o"), TWOSTEP SEIDEL (dotted line with " $x$ "), Sparse VODE (dotted line with " 0 "), Sparse SEULEX (dashed line with " 0 ") and QSSA (dash-dotted line with " $x$ ").
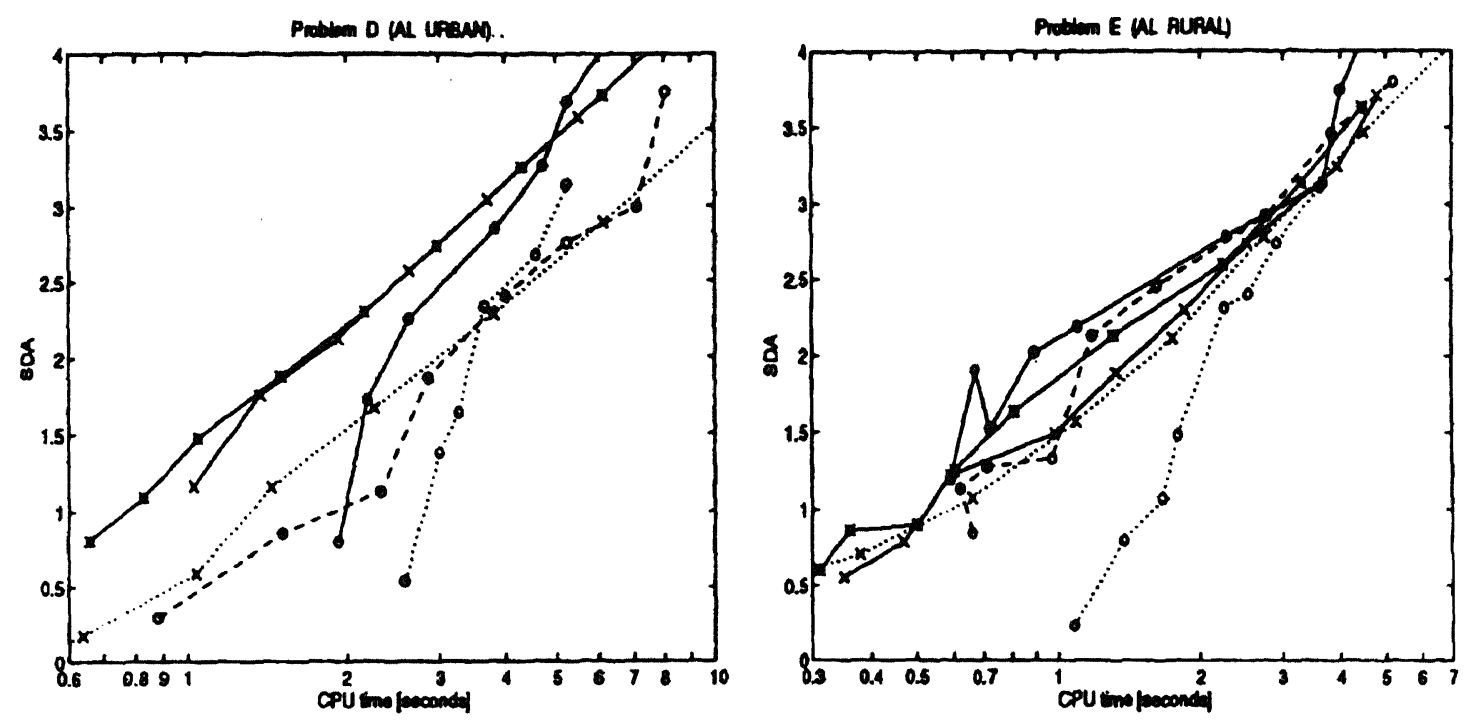

Fig. 4. Work-precision diagram for test problems D and E (AL): Sparse RODAS3 (solid line with "*"), Sparse ROS3 (solid line with " $x$ "), Sparse RODAS (solid line with " 0 "), TwOSTEP SEIDEL (dotted line with " $x$ "), Sparse VODE (dotted line with "o") and Sparse SEULEX (dashed line with " 0 ").

positioning of TWOSTEP (as compared to the CBM-IV cases) is most likely due to the increased number of species in AL.

The results for the urban problem with a $15 \mathrm{~min}$ restart time are presented in Fig. 3. We see more or less the same behaviour relative to the $1 \mathrm{~h}$ restart time as for the CBM-IV problem. Now TWOSTEP has become competitive to RODAS 3 and ROS3 in the $10 \%$ error range, while the curve for RODAS reveals a rather strong non-monotonic accuracy-efficiency behaviour. Again the QSSA solver we previously applied in Sandu et al. (1996b) severely lags behind all others.

\subsection{Problem $F$ : the stratospheric model}

The work-precision diagram given in Fig. 5 again reveals a very good performance of the Rosenbrock solvers compared to the other three. The higher order of accuracy of RODAS is again borne out and again notable is the close performance of ROS3 and RODAS 3 . 


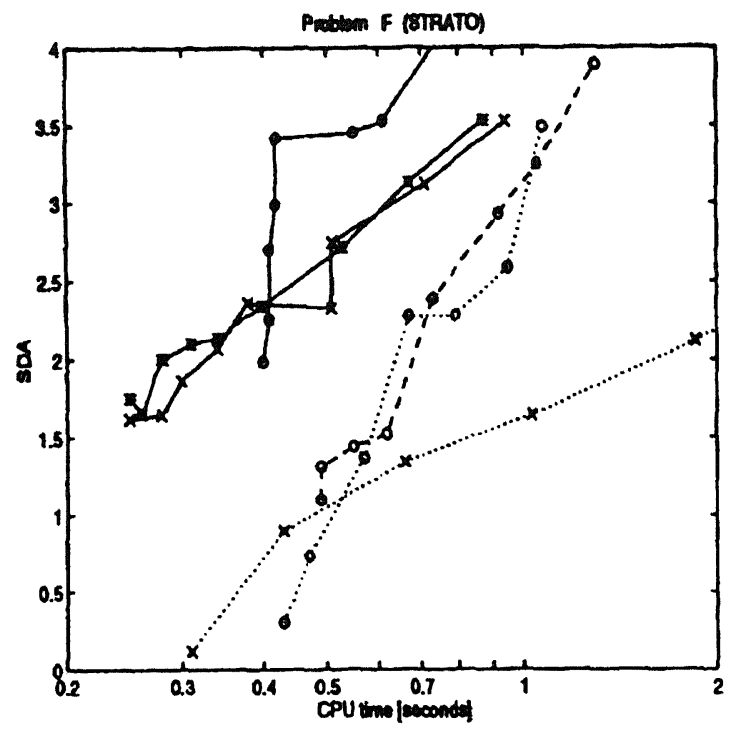

Fig. 5. Work-precision diagram for test problem F (STRATO): Sparse RODAS3 (solid line with "*"), Sparse ROs3 (solid line with " $x$ "), Sparse RODAS (solid line with "o"), TWOSTEP SEIDEL (dotted line with " $x$ "), Sparse VODE (dotted line with "o") and Sparse SEULEX (dashed line with "o").

VODE and SEULEX have similar performance, but are more than 2 times slower than the Rosenbrock codes in the $1 \%$ accuracy range. TWOSTEP follows at a large distance. We should recall, however, that for this problem no emissions were prescribed.

\subsection{Problem $G$ : the aqueous model}

As pointed out in Sandu et al. (1996b) this test problem is the most difficult one from the numerical point of view. The Jacobian $f^{\prime}(y)$ of the derivative function (1) contains stiff eigenvalues for which the relation $\lambda_{i} \approx-L_{i}$ (with $L_{i}$ the destruction term associated with species $i$ ) does not hold. Such eigenvalues are due to the rapid gas-liquid phase interactions and cannot be associated with certain species; for this reason, all the explicit solvers tested in Sandu et al. (1996b) failed to efficiently integrate the AQUEOUS model. As a consequence, in the present work TwOSTEP was not applied to this problem. The results plotted in Fig. 6 for the other solvers are very much in line with those for the stratospheric problem. In the low accuracy range the Rosenbrock family has the lead again, the performances of RODAS, RODAS 3 and ROS3 being very close to each other. SEULEX is about three times slower for 2 accurate digits, but seems to become the best for more than 4 digits; for higher accuracies, VODE changes slope and is not competitive.

\section{OVERALL CONCLUSIONS AND REMARKS}

The answer to the question of which stiff integrator is "the best" for being used in air quality models

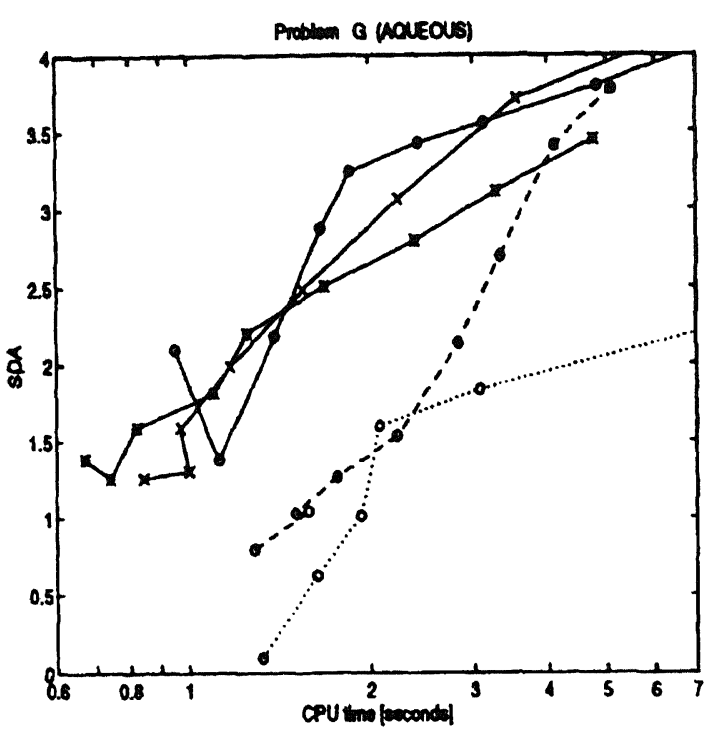

Fig. 6. Work-precision diagram for test problem G (AQUEous): Sparse RODAS3 (solid line with “*”), Sparse ROS3 (solid line with " $x$ "), Sparse RoDas (solid line with "o"), Sparse VODE (dotted line with "o") and Sparse SEULEX (dashed line with "o").

depends on a multitude of factors, some of the most important being the specific chemical mechanism employed, the desired accuracy level and the hardware on which the code runs. In the present work we considered a variety of chemical models, we covered the whole range of accuracy levels of practical interest and tested everything on two machines with completely different architectures. The set of tested codes includes TWOSTEP and sparse versions of the extrapolation code SEULEX, of the state-of-the-art BDF codes LSODE, VODE, the Runge-Kutta-type code SDIRK4 and the Runge-Kutta-Rosenbrock-type codes RODAS, RODAS3, ROS3 and ROS4. We have not considered in this benchmark the widely used BDF code SMvgeAR (Jacobson and Turco, 1994). This code is organized to specifically target a vector machine; running it in scalar mode on box models would lead to less than optimal results. We expect that for box models the performance of SMVGEAR will not differ much from that of sparse LSODE and VODE, as it is based on the first BDF code from Gear (1971). In the numerical ODE literature, this first Gear code has been replaced by the related solvers LSODE and VODE.

Although we have used utmost precaution in implementing the models and in testing the codes, still undiscovered errors and/or less optimal settings of user parameters may have affected part of the numerical results. The interested reader therefore is invited to repeat the experiments using our codes from (CGRER ftp site, 1996) and to join us* in this

* Contact Sandu (sandu@cgrer.uiowa.edu) or Verwer (janv@cwi.nl). 
benchmark activity. The present results give rise to the following main conclusions:

- For all the test problems considered here and within 4 digits of accuracy the Rosenbrock solvers clearly provide the most cost-effective solutions among the codes tested in this paper and in Sandu et al. (1996b).

- The relative ranking between the four sparse Rosenbrock solvers differs per problem, but only to a limited amount. For lower accuracies of practical interest RODAS 3 and ROS3 are usually the best. As expected, for higher accuracies RODAS is mostly competitive; the performance of the solver ROS4 is close to that of RODAS3 and ROS3. In passing we note that our test results do not consistently show that the property of stiff accuracy is truly advantageous for nonlinear problems.

- The above conclusion about the computational speed and accuracy of Rosenbrock methods is also supported by the comparison with the EBI method for Problem A and with the QSSA method for Problems B, D with a $15 \mathrm{~min}$ restart time (the latter has been tested more extensively in Sandu et al., 1996b). Noteworthy is that the QSSA solver lags very far behind in all our experiments.

- Also robustness and ease of use are very important since in actual 3D transport a subtle tuning of the ODE code is cumbersome due to the large variety of conditions that will occur at different grid points. In this respect the Rosenbrock solvers are advocated as well. With the preprocessor KPP at hand, they are easy to use.

- Concerning robustness we have to point out that large values of rtol ( $\geqslant 0.1$ say) combined with too large values for $h_{\min }$ and $h_{\text {start }}$ can cause the Rosenbrock solvers to drift away from the real solution, see Table 2. In these cases the initial transients are not resolved sufficiently accurately. Implicit solvers can also get into trouble here through convergence failures in the iterative modified Newton process. These problems can easily be avoided by choosing $h_{\min }$ and $h_{\text {start }}$ sufficiently small and rtol $\leqslant 0.01$. Since Rosenbrock solvers may increase the step size rapidly, they can remain cost effective even with smaller starting values.

- The extrapolation code SEULEX never ran into a breakdown or returned a negative result. Appar- ently this code works very robust. However, in the low accuracy range SEULEX is always significantly more expensive than RODAS3.

- With regard to robustness, also EBI performs outstanding. The method does not break down when used with a very large step size. We have only applied it to the TMk model we got from Dentener (1996), but our experience is in accordance with that reported in Hertel et al. (1993) and Krol (1996) for different variants of the CBM-IV mechanism. Of course, the main drawback of the EBI approach is that it is intertwined with the chemistry and needs to be adjusted and retested any time the chemistry model is changed. The low accuracy of the solver is mainly due to the use of the first order Euler backward method. Implementation of the EBI approach with a higher order solver (e.g. TWOSTEP) may lead to a notable improvement of accuracy.

- TWOSTEP also performs extremely well with regard to robustness. The entries in Table 2 are due to negative SDA values, rather than breakdowns. This solver can handle both very large step sizes and crude tolerances. It seems to have only one serious limitation, which concerns gas-aqueous phase models. These models do require a linearly or fully implicit solver (Sandu et al., 1996b). Even though in our test problems it lags behind Rosenbrock solvers, TWOSTEP remains, due to its explicit nature, an excellent candidate for very large tropospheric gasphase problems with very small operator split steps. An additional advantage is that the GaussSeidel approach on which TwOSTEP is based, can be effectively extended towards a tridiagonal Gauss-Seidel approach for the coupled solution of chemistry and vertical turbulent diffusion (Verwer et al., 1996a; Spee et al., 1996).

- Often the work-precision curves are non-monotonic, revealing the situation that more CPU time is spent, yet a less accurate solution is obtained. This non-monotonicity is seen mostly for very low tolerances and is caused by the step size selection process (and dynamic iteration strategies in implicit solvers). These work out non-smoothly, as is for example shown very clearly in the diagrams in Hairer and Wanner (1991). Inspection of our diagrams shows that the only variable step size solver yielding monotonic curves in all tests presented is TWOSTEP.

Table 2. The values of rtol for which the codes either break down or give a solution with more than $100 \%$ relative error (negative SDA)

\begin{tabular}{llllll}
\hline Code & B & D & E & Test & G \\
\hline RODAS & $1,0.3,0.1$ & $1,0.3$ & - & - & - \\
RODAS3 & 1 & 1 & - & - & - \\
ROS3 & $1,0.3$ & $1,0.3$ & - & - & $\overline{1}$ \\
TWOSTEP & $1,0.3$ & 1 & - & $0.3,0.1$ & all \\
VODE & 1 & $1,0.3,0.1$ & 1 & $1,0.3$ & 1 \\
\hline
\end{tabular}


- Finally, one word to the interested modellers. In this paper we presented several options not considered before for choosing a chemical solver. As mentioned above, the performance depends on a multitude of factors; thus selecting an integrator should involve testing the most promising codes on the particular application considered. In this context our benchmark results should be thought of as guidelines, but they are no substitute for a careful, problem specific testing.

Acknowledgements-The authors gratefully acknowledge the following organizations for their financial support: A. Sandu, G.R. Carmichael and F.A. Potra were supported by the DOE under grant no DE-FG02-94 ER 61855, J.G. Blom by the Dutch HPCN Program (TASC project HPCN for Environmental Applications), E.J. Spee by the RIVM (Dutch National Institute of Public Health and Environmental Protection, Project CIRK) and J.G. Verwer by Cray Research Inc. via the Stichting Nationale Computer Faciliteiten (National Computing Facilities Foundation, grant CRG 96.03, Project CIRK).

\section{REFERENCES}

Atkinson, R. D., Baulch, D. L., Cox, R. A., Hampson, R. F. J. Kerr, J. A. and Troe, J. (1989) Evaluated kinetic and photochemical data for atmospheric chemistry. J. Chem. Kinetics 21, 115-190.

Brown, P. N., Byrne, G. D. and Hindmarsh, A. C. (1989) VODE: A variable coefficient ODE Solver. SIAM J. Sci. Stat. Comput.. 10, 1038-1051.

Carmichael, G. R., Peters, L. K. and Kitada, T. (1986) $A$ second generation model for regional-scale transport/chemistry/deposition. Atmospheric Environment 20, 173-188.

CGRER (1996) ftp.cgrer.uiowa.edu. Ftp site at the Center for Global and Regional Environmental Research, University of Iowa (cd pub/Ode_benchmark, mget *).

Damian-Iordache, V. and Sandu, A. (1995) KPP - A symbolic preprocessor for chemistry kinetics - User's guide. Technical report, Department of Mathematics, University of Iowa.

Dekker, K. and Verwer, J. G., (1984) Stability of Runge-Kutta Methods for Stiff Nonlinear Differential Equations. North-Holland, Amsterdam.

Dentener, F. (1996) Personal communication, Institute for Marine and Atmospheric Research, University of Utrecht, The Netherlands.

Dentener, F. (1993) Heterogeneous chemistry in the troposphere. Ph.D. thesis, Institute for Marine and Atmospheric Research, University of Utrecht, The Netherlands.

Deufhard, P. (1985) Recent progress in extrapolation methods for ordinary differential equations. SIAM Rev. 27, 505-535.

Dnestrovskaya, E. Yu., Kalitkin, N. N. and Kusmina, L. V. (1994) Lq-decreasing monotonic schemes with complex coefficients and applications to complicated PDEs. Appl. Numer. Math. 15, 327-340.

Gear, C. W. (1971) Numerical Initial Value Problems in Ordinary Differential Equations. Prentice-Hall, Englewood Cliffs, NJ.

Gery, M. W., Whitten, G. Z., Killus, J. P. and Dodge, M. C. (1989) A photochemical kinetics mechanism for urban and regional scale computer modeling. J. geophys. Res., 94, 12,925-12,956.

Hairer, E. and Wanner, G. (1991) Solving Ordinary Differential Equations II. Stiff and Differential-Algebraic Problems. Springer, Berlin.
Heimann, M. (1995) The global atmospheric tracer model TMk. Report No. 10, Deutches Klimarechnenzentrum (DKRZ).

Hindmarsh, A. C. ODEPACK: A systematized collection of ODE solvers. In IMACS Trans. on Scientific Computation, Vol. 1, Scientific Computing, ed. R. S. Stepleman. North-Holland, Amsterdam.

Jacobson, M. Z. and Turco, R. P. (1994) SMVGEAR: A sparse-matrix, vectorized gear code for atmospheric models. Atmospheric Environment 28, 273-284.

Krol, M. (1996) Personal communication, Institute for Marine and Atmospheric Research, University of Utrecht, The Netherlands.

Lurmann, F. W., Loyd, A. C. and Atkinson, R. (1986) A chemical mechanism for use in long-range transport/acid deposition computer modeling. J. geophys. Res. 91, 10,905-10,936.

Hertel, O., Berkowicz, R., Christensen, J. and Hov, Ø. (1993) Test of two numerical schemes for use in atmospheric transport-chemistry models. Atmospheric Environment 27 A. 2591-2611.

Matthijssen, J. (1995) Personal communication, Laboratoire d'Aerologie OMP, Toulouse, France.

Rosenbrock, H. H. (1963) Some general implicit processes for the numerical solution of differential equations. Comput. J. 5, 329-330.

Sandu, A., Potra, F. A., Damian, V. and Carmichael, G. R. (1996a) Efficient implementation of fully implicit methods for atmospheric chemical kinetics. J. Comput. Phys. 129. 101-110.

Sandu, A., Verwer, J. G., van Loon, M., Carmichael, G. R., Potra, F. A., Dabdub, A. and Seinfeld, J. H., (1996b) Benchmarking stiff ODE solvers for atmospheric chemistry problems I: implicit versus explicit. CWI Report NM-R9603 and Report in Computational Mathematics no 85, Department of Mathematics, The University of Iowa. Revised form to appear in Atmospheric Environment.

Simpson, D., Andersson-Sköld, Y. and Jenkin, M. E. (1993) Updating the chemical scheme for the EMEP MSC-W oxidant model: current status. Technical Report 2/93, EMEP MSC-W, The Norwegian Meteorological Institute, Oslo.

Spee, E. J., de Zeeuw, P. M., Verwer, J. G., Blom, J. G. and Hundsdorfer, W. (1996) A numerical study for global atmospheric transport problems. Revision of CWI Report NM-R9620, to appear.

Steihaug, T. and Wolfbrandt, A. (1979) An attempt to avoid exact Jacobian and nonlinear equations in the numerical solution of stiff differential equations. Math. Comp. 33, 521-534.

van Loon, M. (1996) Numerical methods in smog prediction. Ph.D. thesis, University of Amsterdam; (1997) CWI Tract 120. CWI, Amsterdam, The Netherlands.

Verwer, J. G. (1977) S-stability properties for generalized Runge-Kutta methods. Numer. Math. 27, 359-370.

Verwer, J. G. (1994) Gauss-Seidel iterations for stiff ODEs from chemical kinetics. SIAM J. Scientific. Comput. 15, $1243-1250$.

Verwer, J. G. and Scholz, S. (1983) Rosenbrock methods and time-lagged Jacobian matrices. Beiträge zur Numer. Math. 11, 173-183.

Verwer, J. G. and Simpson, D. (1995) Explicit methods for stiff ODEs from atmospheric chemistry. Appl. Numer. Math. 18, 413-430.

Verwer, J. G., Scholz, S., Blom, J. G. and Louter-Nool, M (1983) A class of Runge-Kutta-Rosenbrock methods for solving stiff differential equations. Z. Angew. Math. Mech. 63, 13-20.

Verwer, J. G., Blom, J. G. and Hundsdorfer, W. (1996a) An implicit-explicit approach for atmospheric transport-chemistry problems. Appl. Numer. Math. 20. 191-209. 
Verwer, J. G., Blom, J. G., van Loon, M. and Spee, E. J. (1996b) A comparison of stiff ODE solvers for atmospheric chemistry problems. Atmospheric Environment 30, 49-58.

Zlatev, Z. (1995) Computer Treatment of Large Air Pollution Models. Kluwer Academic Publishers, Dordrechtt.

\section{APPENDIX}

Consistency and stability of Rosenbrock methods

The performance of an integration method largely depends on its order of consistency and its stability properties. Again for the convenience of readers from the atmospheric research community, in this Appendix we will briefly discuss the consistency property for the Rosenbrock method as well as some useful results from the linear stability theory. Also some attention will be paid to the notion of stiffaccuracy.

Consistency conditions. The consistency conditions are found from a formal Taylor expansion of the local error. Le $y_{n+1}=E\left(y_{n}\right)$ be a compact notation for the Rosenbrock method. The difference

$$
\delta_{h}(t)=E(y(t))-y(t+h)
$$

where $y^{\prime}$ is the exact (local) solution of the ODE system $\dot{y}=f(y)$ passing through $y(t)$, is called the local error and the largest integer $p$ for which

$$
\delta_{h}(t)=O\left(h^{p+1}\right), \quad h \rightarrow 0
$$

is called the order of consistency. Hence $\delta_{h}(t)$ is the error after a single step from an exact solution, while the order reveals how rapidly $\delta_{h}(t)$ approaches zero for a decreasing step size. Assuming sufficient differentiability of $y$ and $f$, the order $p$ is determined by Taylor expanding the local error and equating to zero the resulting terms up to the $p$-th one. This leads to the so-called consistency conditions which are expressions in the formula coefficients. Satisfying these conditions gives the desired order $p$. While the expansion is technically complicated and the resulting conditions can become quite lengthy for a large $p$, the derivations are conceptually simple. For a maximum of four stages, the conditions for order $p \leqslant 3$ are

$$
\begin{array}{ll}
p=1: & b_{1}+b_{2}+b_{3}+b_{4}=1 \\
p=2: & b_{2} \beta_{2}^{\prime}+b_{3} \beta_{3}^{\prime}+b_{4} \beta_{4}^{\prime}=\frac{1}{2}-\gamma \\
p=3: & b_{2} \alpha_{2}^{2}+b_{3} x_{3}^{2}+b_{4} \alpha_{4}^{2}=\frac{1}{3} \\
: & b_{3} \beta_{32} \beta_{2}^{\prime}+b_{4}\left(\beta_{42} \beta_{2}^{\prime}+\beta_{43} \beta_{3}^{\prime}\right) \\
& =\frac{1}{6}-\gamma+\gamma^{2}
\end{array}
$$

where

$$
\beta_{i j}=\alpha_{i j}+\gamma_{i j}, \quad \alpha_{i}=\sum_{j=1}^{i-1} \alpha_{i j}, \quad \beta_{i}^{\prime}=\sum_{j=1}^{i-1} \beta_{i j} .
$$

The conditions for $p \leqslant 5$ and general $s$ can be found in Section IV.7 of Hairer and Wanner (1991).

Linear stability. Let $\varepsilon_{n}=y_{n}-y\left(t_{n}\right)$ denote the global error: the difference between the sought exact solution of the ODE system $\dot{y}=f(y)$ and the computed approximation. The global error at the forward time level $t=t_{n+1}$ can be seen to satisfy

$$
\varepsilon_{n+1}=E\left(\varepsilon_{n}+y\left(t_{n}\right)\right)-E\left(y\left(t_{n}\right)\right)+\delta_{n}\left(t_{n}\right)
$$

showing that this error consists of two parts: the local error (7), which is a functional of the exact solution, and the difference

$$
E\left(\varepsilon_{n}+y\left(t_{n}\right)\right)-E\left(y\left(t_{n}\right)\right)
$$

where $E\left(\varepsilon_{n}+y\left(t_{n}\right)\right)$ represents the actual Rosenbrock step taken from the approximation $y_{n}=\varepsilon_{n}+y\left(t_{n}\right)$ and $E\left(y\left(t_{n}\right)\right)$ represents the hypothetical Rosenbrock step taken from the exact solution $y\left(t_{n}\right)$. This difference term reveals a dependence of $\varepsilon_{n+1}$ on $\varepsilon_{n}$. For a proper functioning of the Rosenbrock method it is desirable that, in an appropriate norm \| \|,

$$
\left\|E\left(\varepsilon_{n}+y\left(t_{n}\right)\right)-E\left(y\left(t_{n}\right)\right)\right\| \leqslant\left\|\varepsilon_{n}\right\|
$$

because then the integration is stable in the sense that

$$
\left\|\varepsilon_{n+1}\right\| \leqslant\left\|\varepsilon_{n}\right\|+\left\|\delta_{h}\left(t_{n}\right)\right\| .
$$

This error inequality is elementary, but also fundamental for one-step integration methods. It simply shows that all local errors add up to the global error,

$$
\left\|\varepsilon_{n}\right\| \leqslant \sum_{j=0}^{n-1}\left\|\delta_{h}\left(t_{j}\right)\right\|
$$

if we assume that at the initial time $t_{0}$ the error $\varepsilon_{0}=0$. From inserting $\delta_{h}\left(t_{j}\right)=\mathrm{O}\left(h^{p+1}\right)$, while assuming $h \rightarrow 0$ and $n \rightarrow \propto$ such that $t_{n}=n h$ is fixed, it follows that $\varepsilon_{\mathrm{r}}=\mathrm{O}\left(h^{\mathrm{p}}\right)$. By adding up all local errors one power of $h$ is lost, resulting in a convergence order $p$.

If equation (10) does not hold, the global error can accumulate unboundedly. The integration is then unstable and of no practical use. Whereas for general nonlinear stiff ODEs from chemistry no stability analysis exists for Rosenbrock methods, their stability is well understood for stable, linear systems

$$
\dot{y}=J y
$$

with eigenvalues $\lambda$ satisfying $\operatorname{Re}(\lambda) \leqslant 0$. From practical experience we know that linear stability often provides a satisfactory prediction of stability for nonlinear problems if $J$ is interpreted as the Jacobian matrix $f^{\prime}(y)$. This interpretation is based on a linearization argument, see Dekker et al. (1984) and Hairer and Wanner (1991). Applied to (11), the Rosenbrock method $y_{n+1}=E\left(y_{n}\right)$ reduces to the linear recursion

$$
y_{n+1}=R(h J) y_{n}
$$

where $R(h J)$ is a matrix-valued rational function that approximates the matrix-valued exponential function $\mathrm{e}^{h J}$, being the solution operator of (11). By inserting (12) into the error equation (9), we obtain

$$
\varepsilon_{n+1}=R(h J) \varepsilon_{n}+\delta_{n}\left(t_{n}\right)
$$

or, equivalently,

$$
\varepsilon_{n}=R^{n}(h J) \varepsilon_{0}+\sum_{j=0}^{n-1} R^{n-1-j}(h J) \delta_{h}\left(t_{j}\right)
$$

where, as before, $n=1,2, \ldots$. We see that the demand of stability can now be expressed as boundedness of powers of $R(h J)$, i.e.,

$$
\left\|R^{n}(h J)\right\| \leqslant C
$$

where $C$ is a constant which is independent of $n$ and $h J$. This independence guarantees unconditional stability in the sense that no restrictions exist on the step size. Condition (13) holds if we require that the scalar rational function $R(z)$. 
which is called the stability function, satisfies $|R(z)| \leqslant 1$ for arbitrary $z=h \lambda, \operatorname{Re}(z) \leqslant 0$. This is the famous property of A-stability originally proposed by Dahlquist (see Hairer and Wanner, 1991). We note in passing that for our application we do not really need A-stability, since for atmospheric chemistry the eigenvalues of the Jacobian are always located in the neighbourhood of the real axis. So we actually need the boundedness property only near the negative half line.

We will impose the condition of L-stability, which in addition to A-stability, requires $R(\infty)=0$. L-stability is known to lead to a somewhat more robust approach and better mimics the damping property of $e^{z}$ for $\operatorname{Re}(z) \leqslant 0$. The property of L-stability is easily verified. The stability function $R$ is found by applying the method to the scalar problem $\dot{y}=\lambda y$. This yields a rational function of the form

$$
R(z)=\frac{P(z)}{(1-\gamma z)^{s}}
$$

where $P$ is a polynomial of degree $s^{\prime}, s^{\prime} \leqslant s$, and the degree of $P$ is less than or equal to $s^{\prime}-1$ if the stability function is to be L-stable. Mostly, $s^{\prime}$ is equal to the number of stages $s$, but $s^{\prime}$ can be smaller. In this paper we only consider methods for which $s^{\prime}=s$.

Stability properties of rational functions of the type (14) have been studied extensively. For our purpose the following results are very useful. Suppose that the order of consistency $p$ of the Rosenbrock method is also the order of consistency of $R$, i.e., $P$ is the largest integer for which $R(z)=\mathrm{e}^{z}+$ $\mathrm{O}\left(z^{p+1}\right), z \rightarrow 0$. For L-stable functions we then usually have $p=s$ or $p=s-1$. In both cases $R$ is uniquely determined by $\gamma$. For the case $p=s-1$, L-stability holds for certain intervals for $\gamma$ and if $p=s$ for one particular value of $\gamma$ (see Section IV.6 and Table 6.4 in Hairer and Wanner, 1991). By way of illustration we list the values of $\gamma$ for $1 \leqslant s \leqslant 4$ in Table 1 .

Stiff accuracy. Stiff accuracy is a property related to the Prothero-Robinson model problem

$$
\dot{y}=\lambda(y-\phi(t))+\dot{\phi}(t)
$$

where $\phi$ is some known function. Its solution reads

$$
y(t+h)=\mathrm{e}^{i h}(y(t)-\phi(t))+\phi(t+h)
$$

and if $\operatorname{Re}(\lambda h) \rightarrow-\infty$, the solution $y(t+h) \rightarrow \phi(t+h)$, irrespective the size of $h$. Prothero and Robinson have investigated under which conditions on the formula coefficients, implicit Runge-Kutta solutions mimic this property. Because, then a method can handle this particular transition to infinite stiffness in an accurate manner, which has been the main motivation for this test model (see Dekker and Verwer, 1984; Hairer and Wanner, 1991). They proposed the term stiff accuracy for this phenomenon.

For the current test model, the global error recursion (9) reads

$$
\varepsilon_{n+1}=R(z) \varepsilon_{n}+\delta_{n}\left(t_{n}\right)
$$

where $\delta_{h}\left(t_{n}\right)$ depends in a certain way on $z=h \lambda, h$ and $\phi$. Hairer and Wanner (1991) show, in Section IV.15, that for any consistent Rosenbrock method,

$$
\begin{gathered}
\delta_{h}\left(t_{n}\right)=\mathrm{O}\left(h^{2} / z\right), \text { for } h \rightarrow 0, \quad z \rightarrow \infty, \\
\alpha_{s i}+\gamma_{s i}=b_{i}(i=1, \ldots, s) \text { and } x_{s}=1 .
\end{gathered}
$$

Hence, the desired transition property holds for the local error and because (15) also implies $R(\infty)=0$, this property holds for the global error as well. They therefore call a Rosenbrock method stiffly accurate if (15) holds.

For general nonlinear stiff problems the virtue of stiff accuracy is not so clear. In Hairer and Wanner (1991) it is argued that stiff accuracy is advantageous when solving stiff differential-algebraic systems with a Rosenbrock method (cf. Proposition 3.12, Section VI.3). For ODEs a similar argument exists which goes as follows. Suppose equation (15) holds. A straightforward computation then reveals the following relation between $y_{n+1}$ and the final stage quantities $k_{s}$ and $Y_{s}$

$$
k_{s}=h J y_{n+1}+h f\left(Y_{s}\right)-h J Y_{s} .
$$

Assuming that $J$ is invertible, we may write

$$
y_{n+1}=Y_{s}-(h J)^{-1}\left(h f\left(Y_{s}\right)-k_{s}\right)
$$

which is the result of one modified Newton iteration for the equation

$$
h f(y)-k_{s}=0
$$

using $Y_{\mathrm{s}}$ as starting value. For given $k_{\mathrm{s}}$ this equation can be interpreted as a collocation equation for a numerical solution. Hence, if the property of stiff accuracy holds, if $J$ is invertible and $Y$, a sufficiently good starting guess, then the Rosenbrock solution $y_{n+1}$ is close to a collocation solution. Observe that for linear systems $\dot{y}=J y$ we always have $h J y_{n+1}=k_{s}$ according to equation (16). If the final increment vector $k_{s}$ is close to a true derivative, this collocation property seems recommendable. Other arguments supporting the notion of stiff accuracy for nonlinear problems do not exist as far as we know. 